\title{
Analysis of the clinical characteristics of pregnant women who were hospitalized for more than 7 days after the implementation of the two-child policy
}

\section{Xin Yan}

Peking University Third Hospital https://orcid.org/0000-0003-3104-4893

Yongqing Wang ( $\square$ mddoctor@163.com)

Peking University Third Hospital https://orcid.org/0000-0002-2748-1030

\section{Research article}

Keywords: The two-child policy; Hospital stay; Pregnancy complication; Hospitalization cost

Posted Date: September 16th, 2019

DOI: https://doi.org/10.21203/rs.2.14440/v1

License: (c) (1) This work is licensed under a Creative Commons Attribution 4.0 International License.

Read Full License 


\section{Abstract}

Background: To analyze the clinical characteristics of pregnant women who were hospitalized for more than 7 days after the implementation of the two-child policy. Methods: The patients who were hospitalized for more than 7 days before and after the implementation of the two-child policy were divided into a study group (after the implementation of the two-child policy) and a control group (before the implementation of the two-child policy). Data on the clinical diagnosis, rate of cesarean sections, incidence of severe placenta previa, characteristics of the ICU cases and cost of hospitalization in the two groups were analyzed and compared. Result: The proportion of patients who were hospitalized for more than 7 days after the implementation of the two-child policy increased from $28.09 \%$ to $32.44 \%(P<0.05)$. Regarding older patients, the proportion of those with a cesarean section history increased from $15.92 \%$ to $30.15 \%$; however, the rate of cesarean section decreased from $59.26 \%$ to $54.35 \%(P<0.05)$. Among patients who underwent cesarean sections, the proportion of those with placental previa, postpartum hemorrhage, ART and a cesarean section history increased $(P<0.05)$. Compared with that in the control group, the proportion of patients with severe placenta previa in the study group increased from $3.86 \%$ to $8.73(P<0.05)$. The proportion of patients admitted to the ICU increased from $2.90 \%$ to $4.68 \%(P<0.05)$. Conclusion: Changes in the characteristics of obstetrics inpatients after the implementation of the twochild policy are a challenge for obstetricians. Diagnosis and treatment standardization, a more detailed and comprehensive inpatient management approach, and the rational use of health resources should be promoted.

\section{Background}

On January 1st, 2016, the Chinese government issues the following statement: "The Chinese government encourages a couple to have two children; this is the two-child policy (hereinafter referred to as 'the policy')", which means the "two-child age" is around the corner. According to the China Statistical Yearbook (2017), the number of newborns in China in 2016 increased by approximately 1.31 million compared with the number of newborns in 2015. Due to the increase in the number of older women giving birth and the high rate of cesarean sections in the past, many new features have emerged in the clinical work of obstetrics in China, including the extension of the hospital stay, an increase in pregnancy complications, an increasing use of blood products, an increasing hospitalization cost and other phenomena. For the sake of medical safety and better utilization of clinical resources and to reduce severe pregnancy complications and maternal mortality, we enrolled obstetrical inpatients who were hospitalized for more than 7 days from 2012-2018 in Peking University third Hospital as subjects of this study and analyzed their clinical characteristics.

\section{Data And Methods}

1.1 Data 
Obstetrical inpatients from October 1, 2012, to September 30, 2018, were collected from the HIS system at Peking University Third Hospital. The total number of inpatients during this period was 31,597 , of which 9337 were hospitalized for 7 or more days, accounting for $29.55 \%(9337 / 31,597)$ of patients. On October 1st, 2016, 10 months after the implementation of the policy, the cases were divided into two groups: the study group consisted of 3437 inpatients from October 1st, 2016, to September 30th, 2018, and the control group consisted of 5900 inpatients from October 1st, 2012, to September 30th, 2016. We analyzed the following data: diagnosis, the characteristics of the older inpatients, the characteristics of the inpatients who terminated their pregnancies by cesarean section, the characteristics of the inpatients with severe placenta previa, the characteristics of the ICU inpatients and the hospitalization costs. This study was approved by the Ethics Committee of Peking University third Hospital, Ethics No. (2018) $(419 / 01)$.

\subsection{Methods}

\subsubsection{General data collection}

Data on age, hospital stay, diagnosis, use of blood products, hospitalization costs and other important information were collected.

\subsubsection{Grouping and diagnostic criteria}

\subsubsection{Older pregnant women}

Older pregnant women are generally defined as women aged $\geq 35$ years; internationally, much older pregnant women are defined as women aged $\geq 40$ years or $\geq 45$ years. In this study, the standard for much older women was $\geq 40$ years $₫ 1 \otimes 5 \rrbracket$.

\subsubsection{Severe placenta previa}

Severe placenta previa refers to patients who have placenta previa and whose placenta covers the previous cesarean section incision $₫ 2 \bigotimes$. In addition to the above mentioned inclusion of inpatients with severe placental previa, this study also included some cases of placental adhesion due to a history of multiple factors such as uterine cavity surgery or infection.

\subsubsection{Inpatients admitted to the ICU}

The WHO maternal near miss criteria were used $₫ 3 \rrbracket$. 


\subsubsection{Other Diagnoses}

The diagnosis of gestational diabetes mellitus/pregestational diabetes mellitus (GDM/PGDM)and hypertensive disorder of pregnancy (HDP) were based on a Gynecology and Obstetrics reference(People's Health Publishing House, 9th Edition) $₫ 4 \rrbracket$. Postpartum hemorrhage refers to a bleeding volume $\geq 500 \mathrm{ml}$ during vaginal delivery and $\geq 1000 \mathrm{ml}$ during cesarean delivery within 24 hours after delivery of the fetus \5】.

\subsection{Statistical method}

The data were analyzed with SPSS19.0. The measured data were expressed as the mean \pm standard deviation $(x \pm s)$, and a t-test was used to compare the differences between the two groups. A chi-square test was used to compare the differences in the composition ratios of the two groups, and the difference was statistically significant when $\mathrm{P}<0.05$.

\section{Result}

\subsection{The characteristics of the pregnant women who were hospitalized for more than 7 days before and after the implementation of the policy}

The total number of inpatients before and after the implementation of the policy was 21,003 and 10,594, respectively. The number of inpatients in the study group and the control group was 3437 and 5900 , respectively, accounting for $32.44 \%(3437 / 10,594)$ and $28.09 \%(5900 / 21003)$ of all inpatients. When comparing the two groups, the proportions of older pregnant women, much older pregnant women, inpatients terminating pregnancy by vaginal delivery, inpatients terminating pregnancy by cesarean section, inpatients with severe placenta previa, inpatients using assisted reproductive technology (ART), and inpatients admitted to the ICU and the hospitalization cost were all statistically significant $(P<0.05)$, as shown in Table 1.

There were significant differences in the proportions of GDM/PGDM, hypertensive disorder of pregnancy, placenta previa, postpartum hemorrhage, cesarean section, severe placenta previa and termination of pregnancy by cesarean section between the two groups $(P<0.05)($ Table 2$)$.

\subsection{Characteristics of inpatients terminating pregnancy by cesarean section in the two groups}


In the study group and the control group, the number of inpatients terminating pregnancy by cesarean section was 1751 and 3258, respectively. When the two groups were compared, the average age and the average hospitalization cost were higher in the study group than in the control group. The proportions of older inpatients, much older inpatients, inpatients with hypertensive disorder of pregnancy, inpatients with placenta previa, inpatients with postpartum hemorrhage, inpatients who had undergone ART treatment, inpatients with dangerous placenta previa, and inpatients terminating pregnancy by cesarean section increased significantly in the study group $(P<0.05)$. There were no significant differences in the proportions of inpatients with GDM/PGDM or twin pregnancy between the two groups (Table 3).

\subsection{Characteristics of the patients with severe placenta previa in the two groups}

There were 228 inpatients and 300 inpatients with severe placental previa in the study group and control group, respectively. There were significant differences in the average usage of plasma and of suspended red blood cells between the two groups $(P<0.05)$. There were no significant differences in the average hospitalization cost or the proportion of older or most older inpatients (Table 4).

\subsection{Characteristics of the inpatients admitted to the ICU in the two groups}

There was a significant difference between the two groups in terms of the admission to the ICU due to obstetrical complications, such as severe placenta previa $(P<0.05)($ Table 5$)$.

\section{Discussion}

The characteristics of obstetrical inpatients in the obstetrical department of Peking University third Hospital, a critical care referral center for pregnant women in Beijing, changed after the policy was implemented. The main changes were as follows: the average age of pregnant women rose from 31.86 years to 33.04 years; the proportion of older pregnant women increased from $25.34 \%$ to $37.15 \%$; the proportion of much older pregnant women increased from $4.58 \%$ to $7.45 \%$; and the incidence of pregnancy complications, such as GDM/PGDM, hypertensive disorder of pregnancy, placenta previa, postpartum hemorrhage, cesarean section and dangerous placenta previa, increased in older pregnant women. Reports in the literature $₫ 6 \mathbb{6}$ indicate that advanced-age pregnancy has become increasingly common in both developing and developed countries. Family planning policies, delays in pregnancy planning and the development of assisted reproductive technology have led to an increase in the number of older pregnant women. Bekir Kahveci found that compared with pregnant women aged less than 35 years, older women show increased rates of pregnancy complications, such as hypertensive disorder of pregnancy, GDM/PGDM, and cesarean section. The incidences of placenta previa, postpartum 
hemorrhage, ART, cesarean section and severe placenta previa are also significantly increased compared with those before the implementation of the policy. These factors have led to an increase in critical obstetric inpatients, longer hospital stays and higher hospitalization costs in the short term after the implementation of the policy in China.

\subsection{Rate of cesarean section}

In the study group, the cesarean section rate of older pregnant women rose from $15.92 \%$ to $30.15 \%$, but the total cesarean section rate in the study group decreased from 59.26\% to 54.35\%. An indicated cesarean section can effectively decrease the probability of an adverse pregnancy outcome in women with complications. However, a 2015 World Health Organization statement concluded that a cesarean section rate of more than $10 \%$ does not contribute to a decline in maternal or infant mortality. In contrast, a statement issued by the WHO in 2015 indicates that when the cesarean section rate is higher than 10\%, the operation can lead to more complications and deaths. 8 8 rapidly from the mid-1980s to the 1990s. By the beginning of this century, the cesarean section rate was as high as $70 \%$ in multiple hospitals and even $100 \%$ in specific hospitals $\mathbb{9} \mathbf{9}$. This situation, which is the basis of the obstetrical problems observed after the implementation of the current "policy", has caused concern in the domestic medical profession. With the standardization of obstetrical management and further integration with international standards, the cesarean section rate in China has declined steadily year by year from 2012 to 2016\$10》. With the implementation of the policy, a couple can have two children, and the risk and benefit assessment of cesarean section has changed, which is an important reason for the decrease in the cesarean section rate.

\subsection{Improvement of prognosis in patients with severe placenta previa}

The mean number of hospitalization days decreased from 13.5 days to 11.75 days $(P<0.05)$ for patients with severe placenta previa. There was no difference between the two groups in terms of the mean hospital stay before surgery, but the average hospital stay after surgery decreased from 8 days to 6.66 days $(P<0.05)$. The average usage of plasma decreased from $711.83 \mathrm{ml}$ to $445.61 \mathrm{ml}$, and the average usage of suspended red blood cells decreased from $7.36 U$ to $4.54 U$ in recent years $(P<0.05)$. A history of cesarean section increases the risk of severe placenta previa in the second pregnancy, and generally, the amount of cesarean section hemorrhage in placental accretion cases is more than that in other patients. In recent years, with the increasing incidence of placental accretion, Peking University third Hospital has documented many experiences in the diagnosis and treatment of placental accretion. We use the ultrasound scoring system to predict the type and severity of placental implantation $\otimes 11 \otimes b a s e d$ on the following parameters: the placental location and thickness, whether the retroplacental hypoechoic vocal cords had disappeared, whether the bladder lines are continuous, the placental lacuna 
characteristics, the placental basal blood flow signals, cervical morphology, whether there are blood sinuses in the cervix, and the history of cesarean section. There are 2 points per item, and the total score is calculated. A score $\geq 5$ is used to predict the adhesive type and severe type (including implantation and penetrating type, respectively) of placental accretion. When the score is $\geq 10$, the possibility of a penetrating type of placental accretion is higher than that of the other two types of placental accretion. Prenatal dynamic ultrasound examination combined with MRI provides an important reference value for evaluating the severity of placental accretion. These preoperative preparations are effective measures to reduce intraoperative bleeding and the transfusion of blood products. This approach provides important clinical guidance for preparation before termination of pregnancy. In addition, we use a balloon catheter to temporarily block the blood supply artery, which effectively reduces intraoperative bleeding, maintains clarity of the surgical field and provides an opportunity to preserve the uterus $\triangle 12 \bigotimes$. Placental hemorrhage is greatly reduced by ultrasound diagnosis for high-risk cases, the application of the balloon hemostatic method, and the cooperation of the surgical team. Multidisciplinary collaboration and effective collaboration by the MDT team reduces clinical blood consumption and improves patient prognosis.

\subsection{Establishment of the criteria for admission to the ICU}

The results show that the number of patients with internal and surgical diseases admitted to the ICU is lower than that in the control group, which may be related to the establishment of the MICU (Maternal ICU) in our obstetrics department in recent years. With the increase in pregnancy complications in ICU patients and the shortage of ICU resources, patients with internal and surgical complications who do not need invasive surgery or life support are observed in the MICU. Among the inpatients admitted to the ICU, the proportion of inpatients with pregnancy complications increased from $26.32 \%$ to $43.47 \%(P<0.05)$. The rational use of ICU resources and cooperation of obstetrics and ICU doctors provide a guarantee for the diagnosis and treatment of critical obstetric inpatients. In China, most regions and hospitals do not have specialized obstetrical ICUs, and critical obstetric inpatients are usually admitted to the surgical ICU (Public ICU). Due to the physiological characteristics of pregnant women, cooperation between obstetricians and ICU doctors is needed for the management of these women during the perinatal and peri-operative periods. Considering the shortage of ICU resources, relevant criteria for the transfer of patients to the ICU should be established and would ensure not only the rational use of resources but also medical safety. The WHO defines women who are dying of complications during pregnancy as maternal near miss (MNM) and have developed MNM diagnostic criteria to help identify critical pregnant women $\triangle 3,13 \otimes$. The MNM standard clarifies the related factors leading to maternal mortality from three perspectives, namely, the clinical manifestation, laboratory examination and treatment measures, which are not only helpful to identify critical pregnant women but also helpful to guide the treatment and management of critical pregnant women. In our previous study $₫ 14 \rrbracket$, we used acute physiology and chronic health evaluation II, Marshall's MODS scoring standard and the MNM Diagnostic criteria to evaluate patients admitted to the ICU from 2006 to 2011 in Peking University third Hospital and found 
that the MNM diagnostic criteria can better identify critical pregnant women. The MNM diagnostic criteria are suitable for China's national conditions and have a higher positivity rate.

\subsection{Increase in hospitalization cost}

Our results showed that the average hospitalization cost rose from 8766.40 yuan in the control group to $11,595.58$ yuan in the study group. The average hospitalization cost increased from 10,250.77 yuan to $14,666.33$ yuan for patients undergoing cesarean sections due to pregnancy complications. Although the average hospitalization cost was high for both the patients with severe placenta previa and the patients admitted to the ICU, there was no significant difference between the study group and the control group. According to the Law of Social Insurance of China, maternity insurance is a type of social insurance that is provided by the state and society for the temporary interruption in the woman's participation in the labor force due to the birth of a child. However, some scholars have noted that [15] the scope of reimbursement is narrow and that the rate of reimbursement is too low to meet the needs of social development. Most areas of maternity insurance are reimbursed in accordance with local policies, which means that no matter how much money is spent, patients can only be reimbursed a certain amount of money. In some complicated situations during pregnancy, many more of the expenses will not be reimbursed.

After the implementation of the policy, in addition to the pressure to increase the number of pregnancies, the obstetrical risks resulting from the associated increase in pregnancy complications are also a problem that needs attention. To make better use of medical resources, ensure medical safety and reduce the occurrence of adverse maternal and infant outcomes as much as possible, obstetricians should promote the standardization of clinical diagnosis and treatment and a more detailed management strategy based on to the new characteristics of inpatients under the new policy.

\section{Declarations}

Ethical approvals and consent to participate: This study was approved by the Ethics Committee of Peking University Third Hospital, Ethics No. (2018)(419/01).Written informed consent was obtained from all patients.

Consent for Publication: All authors have approved the manuscript for submission

Availability of data and material: Obstetrical inpatients from October 1, 2012, to September 30, 2018, were collected from the HIS system at Peking University Third Hospital.

Competing interests: The authors declare that they have no conflict of interest.

Funding: The National Key Research and Development Program of China Funding number:2016YFC1000208-4 
Authors' contributions: Xin Yan analyzed and interpreted the patient data regarding the clinical characteristics of pregnant women who were hospitalized for more than 7 days after the implementation of the two-child policy, and was a major contributor in writing the manuscript. Yongqing Wang offerred the study conception and design, was a major contributor in data collection. All authors read and approved the final manuscript.

Acknowledgements: Not applicable.

\section{References}

1.Ogawa Kohei,Urayama Kevin Y,Tanigaki Shinji et al. Association between very advanced maternal age and adverse pregnancy outcomes: a cross sectional Japanese study.[J].BMC Pregnancy Childbirth, 2017, 17: 349 .

2.Na L, Tian Y, Caixia L, et al. Feasibility of Infrarenal Abdominal Aorta Balloon Occlusion in Pernicious Placenta Previa Coexisting with Placenta Accrete[J]. BioMed Research International, 2018, 2018:1-6.

3.Say Lale,Souza João Paulo,Pattinson Robert C et al. Maternal near miss-towards a standard tool for monitoring quality of maternal health care.[J].Best Pract Res Clin Obstet Gynaecol, 2009, 23: 287-96.

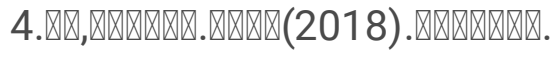

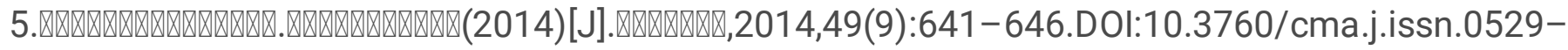
567x.2014.09.001.

6.Wennberg Anna Lena,Opdahl Signe,Bergh Christina et al. Effect of maternal age on maternal and neonatal outcomes after assisted reproductive technology.[J].Fertil. Steril, 2016, 106: 1142-1149.e14.

7.Kahveci Bekir,Melekoglu Rauf,Evruke Ismail Cuneyt et al. The effect of advanced maternal age on perinatal outcomes in nulliparous singleton pregnancies.[J].BMC Pregnancy Childbirth, 2018, 18: 343.

8.World Health Organization. WHO statement on caesarean section rates. Geneva: WHO/RHR/15.02, 2015.

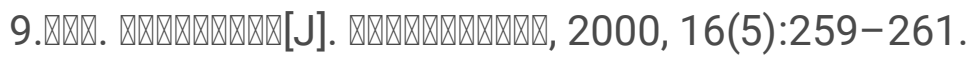

10.Liang Juan,Mu Yi,Li Xiaohong et al. Relaxation of the one child policy and trends in caesarean section rates and birth outcomes in China between 2012 and 2016: observational study of nearly seven million health facility births.[J].BMJ, 2018, 360: k817.

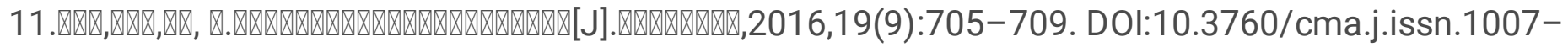
9408.2016.09.014.

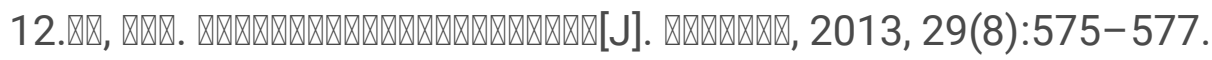


13.Oliveira L C, Costa A A. Maternal near miss in the intensive care unit: clinical and epidemiological aspects[J]. Revista Brasileira De Terapia Intensiva, 2015, 27(3):220.

14.Wang $\mathrm{Y}, \mathrm{Ge} \mathrm{Q}$, Jing W, et al. The WHO near miss criteria are appropriate for admission of critically ill pregnant women to intensive care units in China[J]. Chinese Medical Journal, 2013, 126(5):895-898.

15.

\section{Tables}

Table 1 Characteristics of the inpatients in two groups

\begin{tabular}{ccccc}
\hline & Control group & Study group & $\mathrm{X}^{2} / \mathrm{t}$ & P value \\
\hline Age (years) & 5900 & 3437 & 64.057 & $<0.001$ \\
Older women (n, \%) & $31.857 \pm 4.321$ & $33.043 \pm 4.463$ & 12.526 & $<0.001$ \\
\hline Much older women (n, \%) & $1495(25.34 \%)$ & $1277(37.15 \%)$ & 145.25 & $<0.001$ \\
\hline Vaginal delivery (n, \%) & $270(4.58 \%)$ & $256(7.45 \%)$ & 33.699 & $<0.001$ \\
Cesarean section (n, \%) & $1037(17.58 \%)$ & $672(19.55 \%)$ & 5.669 & 0.017 \\
\hline Severe placenta previa (n, \%) & $2258(55.22 \%)$ & $1751(50.95 \%)$ & 15.960 & $<0.001$ \\
\hline ART (n, \%) & $411(6.97 \%)$ & $401(11.67 \%)$ & 60.447 & $<0.001$ \\
\hline Postpartum hemorrhage (n, \%) & $674(11.42 \%)$ & $569(16.56 \%)$ & 49.554 & $<0.001$ \\
\hline Admitted to ICU (n, \%) & $171(2.90 \%)$ & $161(4.68 \%)$ & 20.202 & $<0.001$ \\
\hline & $8766.395 \pm 17,090.629$ & $11,595.58 \pm 20,550.786$ & 6.814 & $<0.001$ \\
& & & & \\
\hline
\end{tabular}

Table 2 Characteristics of the older inpatients in the two groups 


\begin{tabular}{ccccc}
\hline & Control group & Study group & $\mathrm{X}^{2} / \mathrm{t}$ & P value \\
\hline GDM/PGDM (n, \%) & $542(36.25 \%)$ & $557(43.61 \%)$ & 15.607 & $<0.001$ \\
\hline HDP (n, \%) & $342(22.88 \%)$ & $346(27.09 \%)$ & 6.568 & 0.01 \\
\hline Placenta previa (n, \%) & $176(11.77 \%)$ & $196(15.35 \%)$ & 7.58 & 0.006 \\
\hline Postpartum hemorrhage (n, \%) & $155(10.37 \%)$ & $183(14.33 \%)$ & 10.1 & 0.001 \\
\hline ART (n, \%) & $365(24.41 \%)$ & $288(22.55 \%)$ & 1.326 & 0.25 \\
\hline Cesarean section history (n, \%) & $238(15.92 \%)$ & $385(30.15 \%)$ & 80.03 & $<0.001$ \\
\hline Severe placenta previa (n, \%) & $84(5.62 \%)$ & $121(9.48 \%)$ & 14.957 & $<0.001$ \\
\hline Cesarean section (n, \%) & $886(59.26 \%)$ & $694(54.35 \%)$ & 6.796 & 0.009 \\
\hline
\end{tabular}

Table 3 Characteristics of the inpatients terminating pregnancy by cesarean section in the two groups

\begin{tabular}{ccccc}
\hline & Control group & Study group & $\mathrm{X}^{2} / \mathrm{t}$ & P value \\
\hline Age (years) & $32.159 \pm 4.313$ & $33.379 \pm 4.514$ & 9.263 & $<0.001$ \\
\hline Older women (n, \%) & $889(27.29 \%)$ & $697(39.81 \%)$ & 82.495 & $<0.001$ \\
\hline Much older women (n, \%) & $170(5.22 \%)$ & $148(8.45 \%)$ & 20.039 & $<0.001$ \\
\hline GDM/DM (n, \%) & $1103(33.86 \%)$ & $618(35.29 \%)$ & 1.046 & 0.307 \\
\hline HDP (n, \%) & $755(23.17 \%)$ & $520(29.70 \%)$ & 25.543 & $<0.001$ \\
\hline Placenta previa (n, \%) & $392(12.03 \%)$ & $309(17.65 \%)$ & 29.834 & $<0.001$ \\
\hline Postpartum hemorrhage (n, \%) & $426(13.08 \%)$ & $364(20.79 \%)$ & 50.998 & $<0.001$ \\
\hline ART (n, \%) & $336(10.31 \%)$ & $309(17.65 \%)$ & 54.604 & $<0.001$ \\
\hline Placenta previa (n, \%) & $576(17.68 \%)$ & $559(31.92 \%)$ & 131.875 & $<0.001$ \\
\hline Severe placenta previa (n, \%) & $196(6.02 \%)$ & $244(13.93 \%)$ & 89.135 & $<0.001$ \\
& & & & \\
\hline Twin pregnancy (n, \%) & $605(18.57 \%)$ & $318(18.16 \%)$ & 0.127 & 0.722 \\
\hline
\end{tabular}

Table 4 Characteristics of the patients with severe placenta previa in the two groups 


\begin{tabular}{ccccc}
\hline & Control group & Study group & $\mathrm{X}^{2} / \mathrm{t}$ & $\mathrm{P}$ value \\
\hline Age (years) & $33.09 \pm 4.61$ & $33.49 \pm 4.59$ & 0.99 & 0.323 \\
Older women (n, \%) & $84(36.84 \%)$ & $121(40.33 \%)$ & 0.665 & 0.415 \\
\hline Much older women (n, \%) & $20(8.77 \%)$ & $31(10.33 \%)$ & 0.362 & 0.547 \\
\hline Usage of plasma (ml) & $711.83 \pm 1401.88$ & $445.61 \pm 987.97$ & 2.443 & 0.015 \\
\hline Usage of suspended red blood cells (U) & $7.36 \pm 11.71$ & $4.54 \pm 8.36$ & 3.087 & 0.002 \\
\hline Hospitalization cost (RMB) & $25,399.74 \pm 37069.43$ & $27,421.02 \pm 41782.39$ & 0.587 & 0.557 \\
\hline Hospital stay (days) & $13.50 \pm 8.38$ & $11.75 \pm 6.53$ & 2.608 & 0.009 \\
\hline Hospital stay before surgery (days) & $5.97 \pm 5.30$ & $5.20 \pm 5.66$ & 1.591 & 0.112 \\
\hline Hospital stay after surgery (days) & $8.00 \pm 6.18$ & $6.66 \pm 3.39$ & 2.954 & 0.003 \\
\hline
\end{tabular}

Table 5 Characteristics of the inpatients admitted to the ICU in the two groups

\begin{tabular}{ccccc}
\hline & Control group & Study group & $\mathrm{X}^{2} / \mathrm{t}$ & P value \\
\hline Age (years) & $31.49 \pm 5.26$ & $32.31 \pm 4.80$ & 1.481 & 0.14 \\
\hline Nonpregnancy complications $^{1}(\mathrm{n}, \%)$ & $50(29.24 \%)$ & $32(19.88 \%)$ & 3.91 & 0.048 \\
\hline Pregnancy complications $^{2}(\mathrm{n}, \%)$ & $121(70.76 \%)$ & $129(80.12 \%)$ & 3.91 & 0.048 \\
\hline Severe placenta previa (n, \%) & $45(26.32 \%)$ & $70(43.47 \%)$ & 10.789 & 0.001 \\
\hline ICU stay (days) & $4.81 \pm 8.92$ & $3.49 \pm 4.79$ & 1.693 & 0.092 \\
\hline Hospitalization cost (RMB) & $53,993.63 \pm 83,956.36$ & $61,627.07 \pm 72,764.20$ & 0.887 & 0.376 \\
\hline
\end{tabular}

1. Nonpregnancy complications: heart failure, severe pneumonia, respiratory failure, immune system disease, severe pancreatitis, acute cholecystitis and acute appendicitis, among others.

2. Pregnancy complications: postpartum hemorrhage, severe placenta previa, amniotic fluid embolism, hypertensive disorder of pregnancy, and HELLP syndrome, among others. 